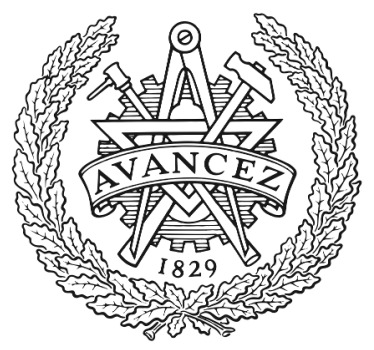

CHALMERS

UNIVERSITY OF TECHNOLOGY

\title{
Logarithmic perturbation models in the weak-dispersion regime with applications to passive optical networks
}

Downloaded from: https://research.chalmers.se, 2023-04-26 13:27 UTC

Citation for the original published paper (version of record):

Oliari, V., Agrell, E., Alvarado, A. (2020). Logarithmic perturbation models in the weak-dispersion regime with applications to passive optical networks. European Conference on Optical Communication, ECOC.

http://dx.doi.org/10.1109/ECOC48923.2020.9333146

N.B. When citing this work, cite the original published paper. 


\title{
Logarithmic Perturbation Models in the Weak-Dispersion Regime with Applications to Passive Optical Networks
}

\author{
Vinícius Oliari(1), Erik Agrell ${ }^{(2)}$, Alex Alvarado ${ }^{(1)}$ \\ (1) Department of Electrical Engineering, Eindhoven University of Technology, Eindhoven, The Nether- \\ lands, v.oliari.couto.dias@tue.nl \\ (2) Department of Electrical Engineering, Chalmers University of Technology, Gothenburg, Sweden
}

\begin{abstract}
Three novel analytical models derived using logarithmic perturbation are presented. The best proposed model achieves a gain of $1.5 \mathrm{~dB}$ when comparing its normalised square deviation with other models in a passive optical network.
\end{abstract}

\section{Introduction}

Perturbation models have been proposed in the literature to approximate the optical fibre propagation, which is governed by the nonlinear Schrödinger equation (NLSE) ${ }^{[1],[2]}$. The NLSE can be described by attenuation, chromatic dispersion and the Kerr effect ${ }^{[3]}$. The Kerr effect is characterised by a nonlinear coefficient, while the chromatic dispersion by the group-velocity dispersion (GVD) parameter. Perturbative models based on the nonlinear coefficient have been widely used in the literature to predict system performance ${ }^{[4],[5]}$ or design low-complexity receivers ${ }^{[6]}$, for example. Recently, we proposed a perturbative model on the GVD parameter ${ }^{[7],[8] \text {. }}$

The most studied perturbative methods in optical fibre communications are the regular perturbation (RP) and the logarithmic perturbation $(L P)^{[1],[9]}$. RP has been presented in the literature for the nonlinear coefficient $\gamma^{[2]}$ and the GVD parameter $\beta_{2}{ }^{[7]}$, while LP has only been performed for $\gamma^{[1],[10]}$. Therefore, in this paper, we present for the first time the LP on $\beta_{2}$ model. The performance of this model, however, can be improved by performing LP in the frequency domain, which is a new approach that we call frequency logarithmic perturbation (FLP). FLP should not be confused with the frequency resolved logarithmic perturbation (FRLP) proposed in ${ }^{[11],[12]}$. FRLP still applies the LP method in the time domain, whereas FLP applies this method in the frequency domain. To the best of our knowledge, this is the first time that FLP models are presented in the literature.

In total, six models arise from the mentioned perturbations. Three of them were already known in the literature: $\mathrm{RP}$ on $\gamma, \mathrm{RP}$ on $\beta_{2}$, and LP on $\gamma$. In this paper, we present three new other models: LP on $\beta_{2}$, FLP on $\gamma$, and FLP on $\beta_{2}$. We compare these six models in terms of normalised square deviation (NSD) in a passive optical network (PON). This system presents low accumulated dispersion due to its short distance, which makes it more suitable for perturbations on the GVD parameter. In addition, the system can operate in the highly nonlinear regime due to high input powers. The results show that FLP on $\beta_{2}$ yields the best performance in the highly nonlinear regime.

\section{Channel Model and Performance Metric}

The normalised NLSE for noiseless propagation of an optical field $E$ for a retarded time frame $t$ and distance $z$ is $^{[2]}$

$$
\begin{aligned}
\partial_{z} A(t, z)= & -\left(j \beta_{2} / 2\right) \partial_{t t} A(t, z) \\
& +j \gamma e^{-\alpha z}|A(t, z)|^{2} A(t, z),
\end{aligned}
$$

where $\alpha$ is the attenuation coefficient. The first term on the right-hand side of (1) represents the chromatic dispersion and the last term represents the Kerr nonlinearity.

A numerically very accurate solution of (1) is obtained by the SSFM and denoted by $A$. We denote the output of a model $M$ by $A_{M}$ and compare it with $A$ in the time domain. The metric used for this comparison is the NSD, defined as ${ }^{[2]}$

$$
\mathrm{NSD} \triangleq \frac{\int_{-\infty}^{\infty}\left|A_{M}(t, z)-A(t, z)\right|^{2} d t}{\int_{-\infty}^{\infty}|A(t, z)|^{2} d t}
$$

The NSD integrates the absolute error squared over the entire propagated time, and normalises it with the power of the signal $A$. The lower the NSD, the more accurate is the analytical model.

\section{Regular Perturbation}

The RP method consists of representing a signal by a power series of a certain coefficient. For the 
first order $\operatorname{RP}$ on $\gamma$, this series is truncated after the first two terms. The resulting first order RP on $\gamma$ model is then given by

$$
A(t, z) \approx A_{\mathrm{RP}}^{(\gamma)}(t, z)=A_{0}^{(\gamma)}(t, z)+\gamma A_{1}^{(\gamma)}(t, z),
$$

where $A_{0}^{(\gamma)}$ and $A_{1}^{(\gamma)}$ represent the zeroth and first order RP on $\gamma$ terms ${ }^{[1],[2]}$. Analogously, the first order RP on $\beta_{2}$ is obtained by truncating the power series of the linear coefficient $\beta_{2}$ after the first two terms. The first order RP on $\beta_{2}$ is written as

$$
A(t, z) \approx A_{\mathrm{RP}}^{\left(\beta_{2}\right)}(t, z)=A_{0}^{\left(\beta_{2}\right)}(t, z)+\beta_{2} A_{1}^{\left(\beta_{2}\right)}(t, z),
$$

where $A_{0}^{\left(\beta_{2}\right)}$ and $A_{1}^{\left(\beta_{2}\right)}$ represent the zeroth and first order RP on $\beta_{2}$ terms $^{[7]}$.

\section{Logarithmic Perturbation}

LP is a mathematical technique similar to RP. LP on $\gamma$ was first presented in ${ }^{[1],[10]}$ and can be shown to have a higher convergence ratio than RP on $\gamma$. LP functions can be obtained directly by the RP functions $A_{k}^{(\gamma)}$ or $A_{k}^{\left(\beta_{2}\right)}$. Following an approach similar to ${ }^{[1]}$, the first order LP on $\gamma$ is written as

$$
A_{\mathrm{LP}}^{(\gamma)}(t, z)=A_{0}^{(\gamma)}(t, z) \exp \left(\gamma \frac{A_{1}^{(\gamma)}(t, z)}{A_{0}^{(\gamma)}(t, z)}\right),
$$

where the terms $A_{0}^{(\gamma)}$ and $A_{1}^{(\gamma)}$ are the RP terms in (3). Since the LP only depends on the RP terms, LP on $\beta_{2}$ can also be derived following the steps $\mathrm{in}^{[1]}$, resulting in

$$
A_{\mathrm{LP}}^{\left(\beta_{2}\right)}(t, z)=A_{0}^{\left(\beta_{2}\right)}(t, z) \exp \left(\beta_{2} \frac{A_{1}^{\left(\beta_{2}\right)}(t, z)}{A_{0}^{\left(\beta_{2}\right)}(t, z)}\right),
$$

where $A_{0}^{\left(\beta_{2}\right)}$ and $A_{1}^{\left(\beta_{2}\right)}$ are the RP terms in (4).

\section{Frequency Logarithmic Perturbation}

The linearity of (3) and (4) with respect to the functions $A_{k}^{(\gamma)}$ and $A_{k}^{\left(\beta_{2}\right)}$, respectively, suggests another approach to obtain a different LP solution. The new approach consists on performing the same steps on the LP derivation presented in $^{[1]}$ in the frequency domain, which we refer to as FLP. By using this approach, we can obtain two new models not yet presented in the literature, namely FLP on $\gamma$ and FLP on $\beta_{2}$. The first order FLP on $\gamma$ is given by

$$
\tilde{A}_{\mathrm{FLP}}^{(\gamma)}(\omega, z)=\tilde{A}_{0}^{(\gamma)}(\omega, z) \exp \left(\gamma \frac{\tilde{A}_{1}^{(\gamma)}(\omega, z)}{\tilde{A}_{0}^{(\gamma)}(\omega, z)}\right),
$$

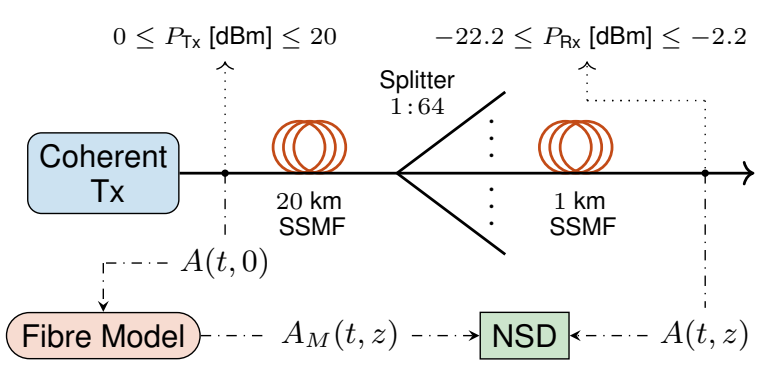

Fig. 1: System setup based on PONs. Due to the short transmission distance, the system has low accumulated dispersion. The range of input powers allow the system to operate in a highly nonlinear regime.

where $\tilde{A}_{0}^{(\gamma)}$ and $\tilde{A}_{1}^{(\gamma)}$ are the Fourier transforms of $A_{0}^{(\gamma)}$ and $A_{1}^{(\gamma)}$ in (3), respectively. The first order FLP on $\beta_{2}$ is given by

$$
\tilde{A}_{\mathrm{FLP}}^{\left(\beta_{2}\right)}(\omega, z)=\tilde{A}_{0}^{\left(\beta_{2}\right)}(\omega, z) \exp \left(\beta_{2} \frac{\tilde{A}_{1}^{\left(\beta_{2}\right)}(\omega, z)}{\tilde{A}_{0}^{\left(\beta_{2}\right)}(\omega, z)}\right),
$$

where $\tilde{A}_{0}^{\left(\beta_{2}\right)}$ and $\tilde{A}_{1}^{\left(\beta_{2}\right)}$ are the Fourier transforms of $A_{0}^{\left(\beta_{2}\right)}$ and $A_{1}^{\left(\beta_{2}\right)}$ in (4), respectively.

\section{Numerical Results}

Fig. 1 shows the PON system setup under consideration. As depicted in the figure, we consider a standard single mode fibre (SSMF) of $20 \mathrm{~km}$, followed by a splitter of ratio $1: 64$ and a final fibre segment of $1 \mathrm{~km}$. With this split ratio, the power budget is $22.2 \mathrm{~dB}$. The fibre input power $P_{\mathrm{Tx}}$ varies from 0 to $20 \mathrm{dBm}$, which leads to a received power $P_{\mathrm{Rx}}$ between -22.2 and $-2.2 \mathrm{dBm}$. The range of powers was chosen to cover launch powers for PON systems according to ${ }^{[13],[14]}$. The SSFM has $\alpha=0.2 \mathrm{~dB} \cdot \mathrm{km}^{-1}, \beta_{2}=-21.67 \mathrm{ps}^{2} \mathrm{~km}^{-1}$, $\gamma=1.2 \mathrm{~W}^{-1} \mathrm{~km}^{-1}$, and operates at a wavelength of $1550 \mathrm{~nm}$ (C-band). The symbol rate for the single transmitted channel was 10 Gbaud and the modulation format quadrature phase shift keying (QPSK). The considered pulse shape was rootraised cosine (RRC), with a roll-off factor of 0.1 . We do not specify any particular receiver, since the study compares the waveforms at the output of the fibre.

To evaluate the impact of the nonlinearities, the NSD was calculated for the powers in the highly nonlinear regime specified in Fig. 1. Fig. 2 shows the NSD versus $P_{\mathrm{Tx}}$ for the six analytical models analysed in this paper. As shown in Fig. 2, both LP and FLP on $\gamma$ outperform RP on $\gamma$. The NSD of FLP on $\gamma$ crosses the $0.1 \%$ NSD threshold at a $P_{\mathrm{Tx}} 1.1 \mathrm{~dB}$ higher than RP on $\gamma$. Analogously, both LP and FLP on $\beta_{2}$ outperform RP on $\beta_{2}$. These results support that, either in the time or 


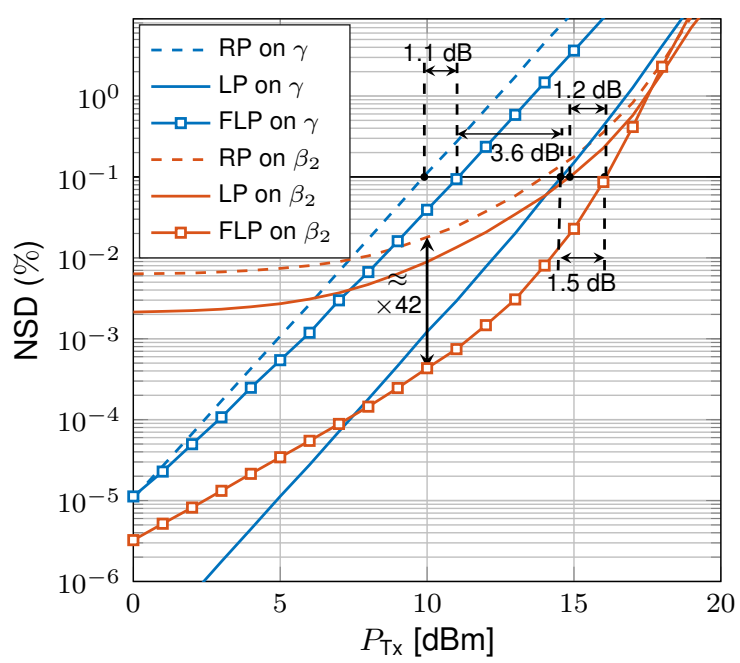

Fig. 2: NSD versus input power $P_{\mathrm{Tx}}$ for the six analytical models presented in this paper. The transmission is performed in C-band. A schematic of the system is shown in Fig. 1.

in the frequency domain, an (F)LP approach converges faster to the true solution of (1) than the standard RP approach.

The results in Fig. 2 also show that LP on $\gamma$ is more accurate than FLP on $\gamma$, while FLP on $\beta_{2}$ is more accurate than LP on $\beta_{2}$. For example, the NSD of LP on $\gamma$ crosses the $0.1 \%$ NSD threshold at a $P_{\mathrm{Tx}} 3.6 \mathrm{~dB}$ higher than FLP on $\gamma$, while that of FLP on $\beta_{2}$ crosses the same threshold at a $P_{\mathrm{Tx}} 1.2 \mathrm{~dB}$ higher than LP on $\beta_{2}$. We believe that the difference between $\beta_{2}$ and $\gamma$ when comparing LP and FLP could be explained by the solution of (1) for only the chromatic dispersion effect or only the Kerr effect ${ }^{[3]}$. The solution for the chromatic dispersion effect only is an exponential in the frequency domain, which resembles the FLP approach. Similarly, the solution for the Kerr effect only is an exponential in the time domain, which resembles the LP approach.

From the six models, FLP on $\beta_{2}$ is the one that presents best performance for $P_{\mathrm{Tx}}$ higher or equal $8 \mathrm{dBm}$. This result suggests that FLP on $\beta_{2}$ is more accurate for weak dispersion and high nonlinearity. For a constant NSD of $0.1 \%$, FLP on $\beta_{2}$ shows a gain of $1.5 \mathrm{~dB}$ in terms of input power when compared to LP on $\gamma$. Larger gains are observed at lower NSDs.

The effects of the dispersion on the models' performance was also investigated. When reducing the accumulated dispersion, the models LP on $\gamma$, RP on $\beta_{2}$, LP on $\beta_{2}$, and FLP on $\beta_{2}$ converge to the true solution of (1). This convergence can be analytically proved by letting $\beta_{2} \rightarrow 0$ in (4), (5), (6), and (8). However, their rate of convergence is different. Fig. 3 shows the NSD versus $\left|\beta_{2}\right|$ for

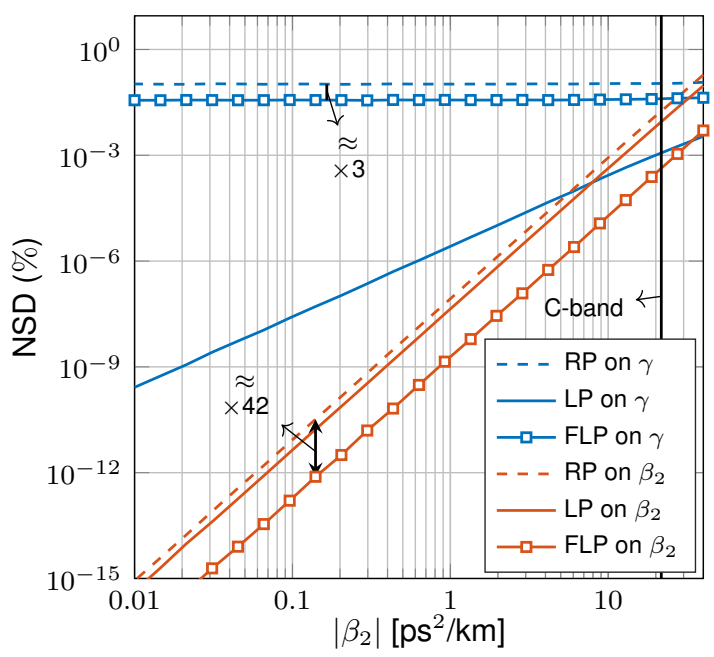

Fig. 3: NSD versus $\left|\beta_{2}\right|$ for the six analytical models presented in this paper. The input power is fixed at $10 \mathrm{dBm}$ for the system shown in Fig. 1 with negative $\beta_{2}$. The models present different rates of convergence when $\beta_{2}$ approachs to zero.

the six analysed models at $P_{\mathrm{Tx}}=10 \mathrm{dBm}$ and negative $\beta_{2}$. As depicted in Fig. $3, \mathrm{RP}$ on $\beta_{2}$, LP on $\beta_{2}$, and FLP on $\beta_{2}$ present a rate of convergence higher than LP on $\gamma$. While the NSD of the three former models increases by approximately $10^{4}$ per decade, that of NSD for LP on $\gamma$ increases by only $10^{2}$ per decade. The NSD for FLP on $\beta_{2}$ is approximately 42 times lower than for $\mathrm{RP}$ on $\beta_{2}$ (see also Fig. 2). This gain is kept approximately constant for other values of $\beta_{2}$. Only FLP on $\gamma$ and RP on $\gamma$ have approximately constant NSD over the shown range of $\beta_{2}$. The NSD for FLP on $\gamma$ was roughly 3 times lower than the NSD for RP on $\gamma$.

\section{Conclusions}

Three novel models based on logarithmic perturbation theory were presented. The validity of the models was investigated in the C-band for a passive optical network. The frequency logarithmic perturbation on $\beta_{2}$ model demonstrated higher accuracy in the highly nonlinear regime than the other models analysed in the paper. Building a receiver based on the proposed model and finding higher order perturbations are possible extensions of this work.

\section{Acknowledgements}

This work is supported by the Netherlands Organisation for Scientific Research (NWO) via the VIDI Grant ICONIC (project number 15685). The work of A. Alvarado has received funding from the European Research Council (ERC) under the European Union's Horizon 2020 research and innovation programme (grant agreement No 757791). The work of E. Agrell has received funding from the Swedish Research Council (VR) under Grant no. 2017-03702. 


\section{References}

[1] E. Forestieri and M. Secondini, "Solving the nonlinear Schrödinger equation", in Optical Communication Theory and Techniques, E. Forestieri, Ed., 1st ed., Boston: Springer US, 2005, pp. 3-11.

[2] A. Vannucci, P. Serena, and A. Bononi, "The RP method: a new tool for the iterative solution of the nonlinear Schödinger equation", J. Lightwave Technol., vol. 20, no. 7, pp. 1102-1112, Jul. 2002.

[3] G. Agrawal, Nonlinear Fiber Optics, 5th, ser. Optics and Photonics. Boston: Academic Press, 2013, pp. 27-56.

[4] A. Ghazisaeidi, "A theory of nonlinear interactions between signal and amplified spontaneous emission noise in coherent wavelength division multiplexed systems", J. Lightwave Technol., vol. 35, no. 23, pp. 51505175, Dec. 2017.

[5] P. Poggiolini, G. Bosco, A. Carena, V. Curri, Y. Jiang, and F. Forghieri, "The GN-model of fiber non-linear propagation and its applications", J. Lightwave Technol., vol. 32, no. 4, pp. 694-721, 2014.

[6] W. Yan, Z. Tao, L. Dou, L. Li, S. Oda, T. Tanimura, T. Hoshida, and J. C. Rasmussen, "Low complexity digital perturbation back-propagation", in European Conference and Exhibition on Optical Communication (ECOC), Sep. 2011.

[7] V. Oliari, E. Agrell, and A. Alvarado, "Regular perturbation on the group-velocity dispersion parameter for nonlinear fibre-optical communications", Nature Communications, vol. 11, no. 933, pp. 1-11, Feb. 2020.

[8] _ - "Regular perturbation for the weak-dispersion regime", in 2019 21st International Conference on Transparent Optical Networks (ICTON), Jul. 2019, pp. 1-4.

[9] M. Secondini, E. Forestieri, and C. R. Menyuk, "A combined regular-logarithmic perturbation method for signal-noise interaction in amplified optical systems", J. Lightwave Technol., vol. 27, no. 16, pp. 3358-3369, 2009.

[10] E. Ciaramella and E. Forestieri, "Analytical approximation of nonlinear distortions", IEEE Photonics Technol. Lett., vol. 17, no. 1, pp. 91-93, Jan. 2005.

[11] M. Secondini and E. Forestieri, "Analytical fiber-optic channel model in the presence of cross-phase modulation", IEEE Photonics Technol. Lett., vol. 24, no. 22, pp. 2016-2019, Sep. 2012.

[12] M. Secondini, E. Forestieri, and G. Prati, "Achievable information rate in nonlinear WDM fiber-optic systems with arbitrary modulation formats and dispersion maps", J. Lightwave Technol., vol. 31, no. 23, pp. 38393852, Nov. 2013.

[13] 10-Gigabit-capable passive optical networks (XG$P O N)$ : Physical media dependent (PMD) layer specification, ITU-T G.987.2, 2016.

[14] PON transmission technologies above $10 \mathrm{~Gb} / \mathrm{s}$ per wavelength, ITU-T G.Sup64, 2018. 